\title{
FRONTIERS OF RF PHOTOINJECTORS
}

\author{
Massimo Ferrario, INFN-LNF Frascati (Rome), Italy
}

\begin{abstract}
New ideas have been recently proposed to achieve ultra-high brightness electron beams, as particularly needed in SASE-FEL experiments, and to produce flat beams, as required in linear colliders. By proper radial shaping of the photoemission laser pulses, a pancake bunch can be created that will evolve automatically into a uniformly filled 3D ellipsoid, i.e. into the ideal bunch. Low emittance schemes already foreseen for split normal conducting photoinjectors have been applied to the superconducting case in order to produce high peak and high average beam brightness. RF compressor techniques have been partially confirmed by experimental results. Research and experiments in the flat beam production from a photoinjector as a possible alternative to damping rings are in progress. An overview of recent advancements and future perspectives in photoinjector beam physics is reported in this paper.
\end{abstract}

\section{INTRODUCTION}

The research and development of high brightness (high current, low emittance) beam production by photoinjectors has been driven in the last decade mainly by self amplified, spontaneous emission, free-electron laser (SASE FEL) applications. Beams with normalized emittances lower than $1 \mu \mathrm{m}$, with peak current of some $\mathrm{kA}$, are required for example for the new x-ray SASE FEL projects $[1,2]$. Usually, to enhance brightness one has to expose emitting cathode to a very high electric field, and also to introduce magnetic solenoid fields within the photoinjector gun region. These focusing fields allow control and mitigation of space-charge effects, a process termed emittance compensation [3]. In the following sections we describe how a beam can be manipulated in straightforward ways to obtain high peack and average brightness in FELs, and the possibility of high luminosity in linear colliders.

In the FEL context a beam dynamics regime of RF photoinjectors, dealing with a violent bunch elongation under the action of longitudinal space charge forces, the Blow-Out regime, has been recently re-considered as a possible alternative to laser pulse shaping techniques $[4,5,6]$. A revival of longitudinal focusing techniques with a deeper understanding of emittance compensation theory [7] has opened up a new possibility of compressing the beam inside an RF structure or in a downstream drift, with a proper beam control employed through solenoid focusing to avoid the emittance degradation [8]. This option may avoid the serious phase space degradations observed in magnetic chicanes caused by coherent synchrotron radiation (CSR) emitted in the bends [9]. Kilo-ampere beams with low emittance have been predicted by simulations for the so-called velocity bunching configuration [8]. At DUVFEL (BNL) [10],
Tokyo University (UTNL) [11], and PLEIADS (LLNL) [12] preliminary experimental results (in non-optimized beam lines) have verified the usefulness of this idea for strongly compressing photoinjector-derived beams, despite the space charge induced emittance degradation observed.

With the advent of proposed superconducting energy recovery linacs (ERL) dedicated to production of radiation [13] that operate at high average current (high duty factor), the demand for high peak and high average brightness, pushes the injector community to consider also the possibility of using a superconducting RF photoinjector.

In a different context, a new technique has been proposed [14] and recently tested [15] at FNAL, the socalled flat beam production, which is an important goal for linear colliders. It consists in a simple transformation of a magnetized round beam, with equal emittances in both transverse planes, produced by a photo-cathode embedded in a solenoid field, which is then followed by a skew quadrupoles triplet. With proper matching, a flat beam with high transverse emittance ratio (300) as required by linear colliders, may in principle be obtained. Experimental results have achieved so far an emittance ratio of 100 .

\section{HIGH BRIGHTNESS BEAM PRODUCTION}

A uniformly filled 3D ellipsoid, the so called "waterbag", is the ideal bunch [16]. The waterbag is characterized by perfectly linear behavior: it is the only charged particle distribution whose internal space charge force field is a linear function of position. The generation of such a bunch configuration directly from the cathode has been recently investigated and its excellent features in terms of negligible emittance growth has been verified by numerical simulations in a realistic photoinjector beam line [17]. Unfortunately the generation of a 3D ellipsoid with uniform charge distribution directly from the cathode is not an easy task and demands challenging solution of the laser pulse shaping system. The techniques proposed in [17] foresee the stacking of a number of Gaussian pulses of different radii or a spectral control of UV beam using four-gratings with masking array in dispersive environment. The demonstration of feasibility of such a laser system is the main challenge for this scheme.

Another way to produce waterbag like bunches has been also recently re-considered [5]. The use of femtosecond photoemission laser pulses in high-gradient RF photoinjectors enables the production of electron bunches whose rest-frame bunch length is much smaller than the bunch radius, the so-called 'pancake' bunch. By proper radial shaping of the photoemission laser pulses a pancake bunch can be created that will evolve 
automatically into a uniformly filled 3D ellipsoid, the so called "blow-out" regime [4]. The clear advantage of this scheme is that longitudinal laser pulse shaping is not anymore an issue, in addition once the beam has reached its final waterbag configuration at the exit of the gun the invariant envelope [7] matching conditions with the booster still hold and the emittance compensation process can be accomplished, as reported in [6]. On the other hand high charge operation $(\sim n C)$ becomes difficult, because image charge on the cathode may drive incorrect final state. The simulation result [5] shown in Fig 1 is the projection in the $\mathrm{x}-\mathrm{z}$ plane at the exit of the gun. The cathode was illuminated with a 100 fs long pulse with 1 $\mathrm{mm}$ spot size and the extracted charge was $100 \mathrm{pC}$. The current profile is close to the parabolic shape expected for a uniform ellipsoidal distribution, Fig $2 \mathrm{a}$, and the distribution in the longitudinal phase space is characterized by perfectly linear behavior, Fig $2 b$.

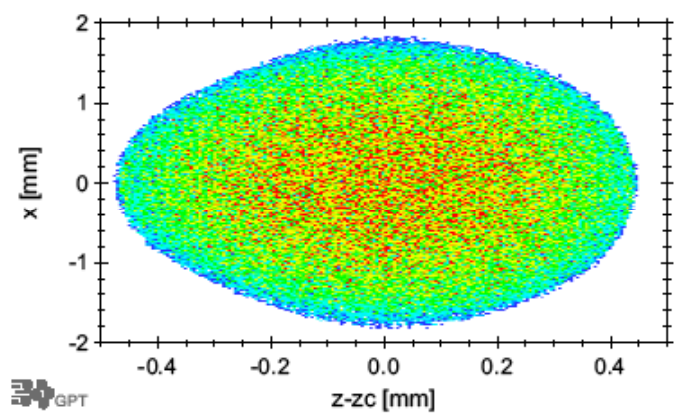

Fig. 1: Projection in the $x-z$ plane of the electron bunch at the exit of the gun.

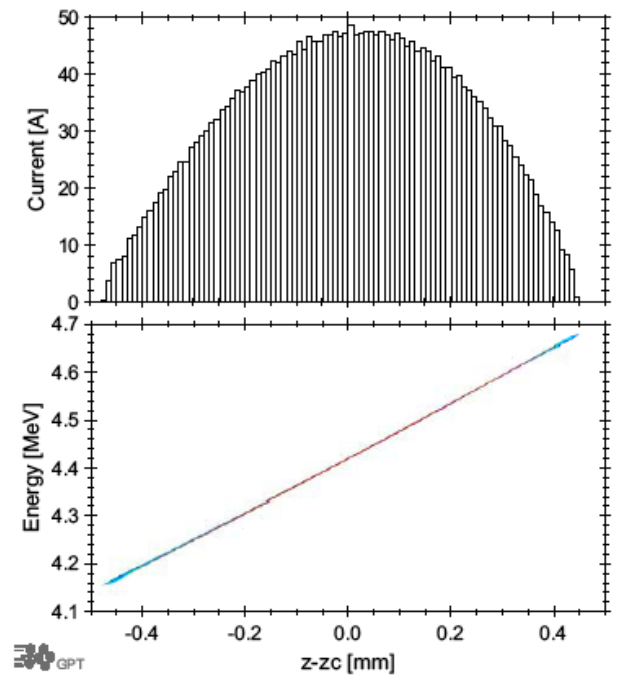

Fig. 2: a) upper plot: current profile b) lower plot: longitudinal phase space distribution of the bunch.

Very interesting progress have been reported concerning the generation of a "beer can" bunch with uniform charge distribution. The laser system equipped with a Dazzler crystal described in [18] will soon allow the generation of a flat top laser pulse with sub ps rise time. An important prerequisite for the optimal operation of the photoinjector working point described in [19], adopted by many photoinjector projects like LCLS, TESLA X-FEL, SPARC [20]. A preliminary confirmation of the usefulness of such a working point has been demonstrated at the Tesla Test Facilty, where a $1 \mathrm{nC}$ beam with longitudinal Gaussian profile has been transported up to the exit of the first cryomodule at $100 \mathrm{MeV}$, i. e. above the space charge dominated beam threshold, with a measured normalized emittance of $2 \mu \mathrm{m}$ in very good agreement with the simulations predictions [21]. A very remarkable results considering that a flat top pulse, when available, should reduce the emittance by a factor 2 , meeting the requirements for the X FEL injector. In the next fall a dedicated experiment will be performed at the SPARC photoinjector to investigate in more details the emittance compensation process by means of a movable emittance meter device [22], in order to fully characterise the $6 \mathrm{D}$ phase space of this working point.

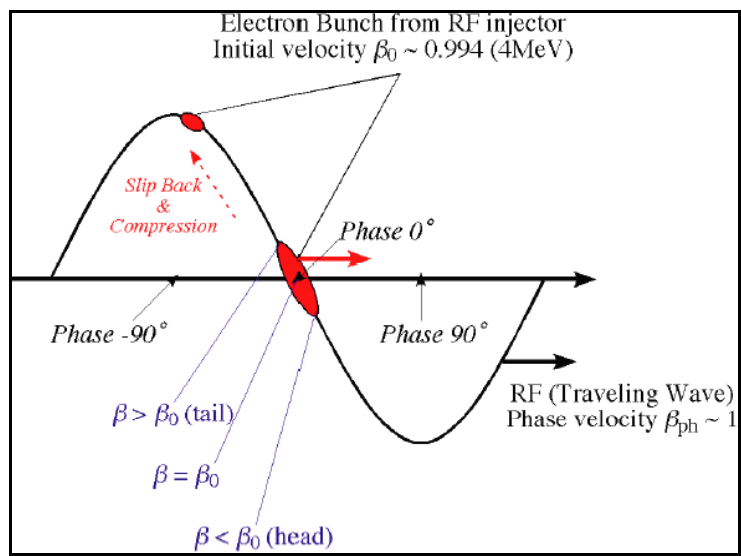

Fig. 3: Scheme of the velocity bunching mechanism.

Since the impact of magnetic compressors on the beam quality is a relevant and compelling topic, with the tendency to have serious emittance growth due to coherent synchrotron radiation effects in bends, a new method able to compress the bunch at moderate energies (tens of $\mathrm{MeV}$ ), using rectilinear trajectories, and integrated in the emittance compensation process, has been proposed [8]. This scheme, see Fig. 3, named velocity bunching, has the following characteristics: although the phase space rotation in this process is still based on a correlated velocity chirp in the electron bunch, in such a way that electrons on the tail of the bunch are faster (more energetic) than electrons in the bunch head, this rotation does not happen in free space but inside the longitudinal potential of a traveling RF wave which accelerates the beam inside a long multi-cell traveling wave (TW) RF structure, applying at the same time an off crest energy chirp to the injected beam. This is possible if the injected beam is slightly slower than the phase velocity of the RF wave so that, when injected at the 
crossing field phase (no field applied), it will slip back to phases where the field is accelerating, but at the same time it will be chirped and compressed. The key point is that compression and acceleration take place at the same time within the same linac section, actually the first section following the gun, that typically accelerates the beam, under these conditions, from a few $\mathrm{MeV}(>4)$ up to 25-35 MeV.

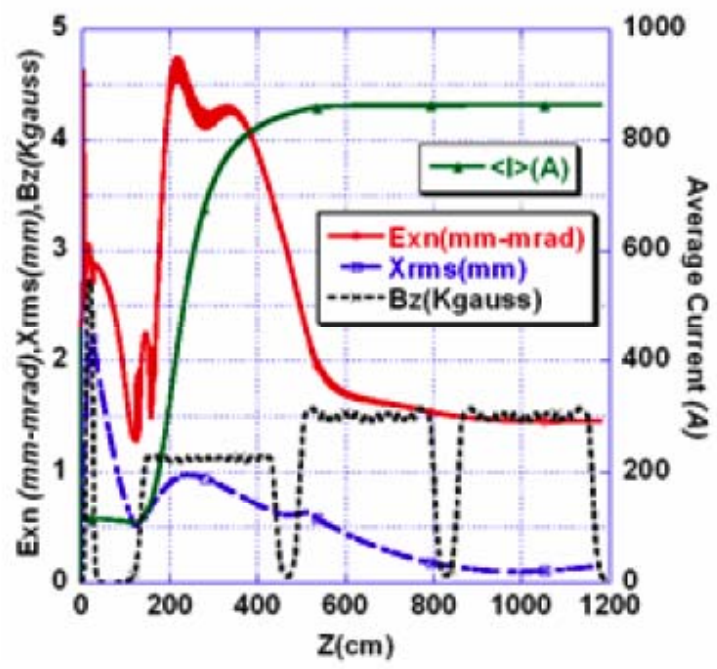

Fig. 4: Average current, transverse emittance and envelope, axial magnetic field vs $\mathrm{z}$ for a 860 A compressed beam.

A careful optimization of a high brightness photoinjector, with integrated velocity bunching as required for the SPARX project has been reported in [23]. The plots in Fig. 4, of the peak current and the transverse normalized rms emittance (a thermal emittance of $0.3 \mathrm{~mm}$ is included) as a function of the distance from the cathode computed by PARMELA for $100 \mathrm{~K}$ particles, show that a peak current of 860 A can be reached with a transverse rms normalized emittance of $1.5 \mu \mathrm{m}$. The final beam energy is only $150 \mathrm{MeV}$, so additional care must still be taken after this point in order to properly damp residual emittance oscillation driven by space charge correlations. The integration of a velocity bunching in a long linac (1 $\mathrm{GeV}$ ) with additional magnetic compression has been also investigated [24] showing the excellent performace of such an hybrid scheme. The experimental results achieved so far are compared in Tab 1. It is important to note that none of the velocity bunching experiment has been performed on a machine specifically designed for this kind of process: the DUV-Fel experiment was performed in a sufficiently high energy linac but without solenoid around the accelerating structures so that emittance control during bunching was impossible. In the Tokyo University experiment the accelerating structure was equipped with the solenoid but the final energy was too low for such an high charge density bunch, nevertheless they have demonstrated the compression for a high charge beam and pointed out the sensitivity to phase jitters. Only the LLNL layout was close to the optimized case and in fact they succeed to demonstrate the possibility to compensate emittance while bunching, down to the level of the non-compressed beam. Anyway the final results in terms of emittance $(10 \mu \mathrm{m})$ is not yet satisfactory. A fully optimized dedicated photo-injector for application of the velocity bunching technique still does not exist: one of the missions of the SPARC project is indeed to design and commission such a system

Table 1: Velocity bunching experiments

\begin{tabular}{|l|l|l|l|l|l|}
\hline & BNL & UCLA & $\begin{array}{l}\text { DUV } \\
\text {-FEL }\end{array}$ & UTNL & LLNL \\
\hline $\begin{array}{l}\text { Charge } \\
\text { [nC] }\end{array}$ & 0.04 & 0.2 & 0.2 & 1 & 0.2 \\
\hline $\begin{array}{l}\text { Length } \\
\text { [ps }]\end{array}$ & 0.37 & 0.39 & 0.5 & 0.5 & $<0.3$ \\
\hline C.R. & 6 & 15 & $>3$ & $>13$ & 10 \\
\hline Reference & {$[25]$} & {$[12]$} & {$[10]$} & {$[11]$} & {$[12]$} \\
\hline
\end{tabular}

\section{SUPERCONDUCTING RF GUN}

In the past, for an implementation of SRF guns it was always assumed that one needs strong focusing inside the gun, near the photocathode. This assumption has been partially driven by relatively low achievable gradient in SRF guns. An interesting solution which avoids use of solenoid fields in transverse beam control near the cathode, so-called "rf focusing", has been proposed in [26]. Unfortunately this method requires a deformation of the cathode plane, causing nonlinear field perturbations that may cause significant emittance growth in the injector. The improvement in superconducting cavity fabrication allows today accelerating gradient higher than $30 \mathrm{MV} / \mathrm{m}$, corresponding to peak field of $60 \mathrm{MV} / \mathrm{m}$ [27]. An alternative scheme in which $\mathrm{rf}$ focusing is not required can today be considered.

A very attractive approach has been proposed at BNL [28]. The basic idea is to illuminate with UV laser the back wall of the superconducting $\mathrm{Nb}$ cavity accelerating in this way photo-emitted electrons. An optimized configuration has been proposed in [29], in which the working point described in [19] has been scaled also to an L-band SC gun design.

This configuration shown in Fig. 5 has a focusing solenoid geometry that keeps most of the magnetic field outside the cavity. In fact in the superconducting case the magnetic field must not penetrate the super-conducting cavity, to avoid thermal breaks down when the critical field of $200 \mathrm{mT}$ is exceeded. The residual fringing field at the level of few Gauss is tolerable in that the focusing is applied only after cool down and the small field is excluded from the superconducting cavity through the Meissner effect, thus avoiding any residual flux trapping that may cause cavity Q0 degradation. In such a design one can obtained the same performance of a normal 
conducting gun with the additional option to work in CW operation, and hence to obtain high average current.

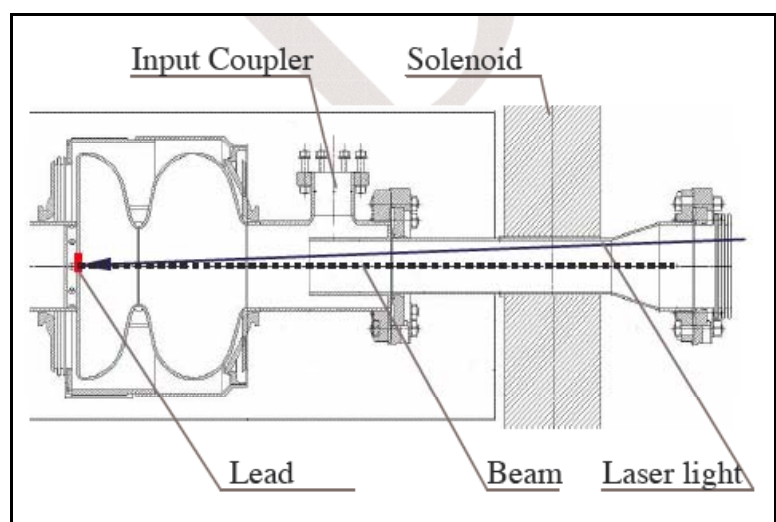

Fig. 5: SC RF gun with Lead cathode.

Another proposed scheme is to excite a TE magnetic mode inside the cavity that focuses the electron beam and prevents the increase of the transverse emittance [30]. Operation with high average beam current requires photocathodes having enhanced quantum efficiency $(\eta)$. When superconductor is used as a photoemitter, high $\eta$ minimizes the thermal load on the superconducting surface. More generally, high $\eta$ implies that one may keep the size and cost of the high duty cycle laser system used to illuminate the photocathode within reasonable limits. It has been recently proposed to use lead as a photocathode deposited on the back wall of the gun cavity. Preliminary measurement [32] show a $\eta=1.710^{-3}$ for a lead cathode illuminated by a $213 \mathrm{~nm}$ laser. With such a good $\eta$ a $3 \mathrm{~W}$ laser could generate 1 $\mathrm{nC}$ beam at $1 \mathrm{MHz}$.

The extreme case of ampere class superconducting guns is discussed in [31]. These devices require careful control of the higher order mode trapping and are specifically designed with wide beam tubes so to facilitate damping of unwanted trapped HOM.

\section{FLAT BEAM PRODUCTION}

In the context of electron-positron linear collider (LC) projects, the goals of electron sources are even more challenging. Linear colliders in fact require high charge, polarized electron beams with extremely low normalized emittances, with geometric average $\sqrt{\varepsilon_{n x} \cdot \varepsilon_{n y}} \approx 10^{-1} \mu \mathrm{m}$. The recent analytical and numerical efforts in understanding beam dynamics in RF photo-injectors have again raised the question whether the performance of an $\mathrm{RF}$ electron gun based injector could be competitive with respect to a damping ring. An injector for a linear collider must provide a flat beam in order to reduce beamstrahlung effects at the interaction point, thus implying $\varepsilon_{n, y}<<\varepsilon_{n, x}$. But a production of a flat beam directly from the cathode surface would increase the difficulties for emittance compensation, easily achieved by means of a symmetric solenoid. A flat beam is typically delivered by a damping ring. Nevertheless a transformation of a round beam derived from a photoinjector into a flat beam has been recently proposed [33] by means of simple linear beam optics adapter at the exit of a injector.

This transformation is possible with a magnetized beam, as produced by an rf gun with a cathode embedded in a solenoid field [14]. At the exit of the gun/solenoid system the beam has an angular momentum given by $p_{\vartheta}=\frac{1}{2} e B_{z, c} R_{c}^{2}$ where $B_{z, c}$ is the on cathode magnetic field, and $R_{c}$ the laser spot radius. Both transverse planes are thus coupled by the beam rotation. Such rotation can be arrested by a suitable choice of a skew quadrupole triplet that, in addition, changes the emittance ratio according to the relation,

$$
\frac{\varepsilon_{x}}{\varepsilon_{y}}=1+\frac{2 \sigma_{r}^{2}}{\beta^{2} \sigma_{r^{\prime}}^{2}}
$$

for a beam with rms size $\sigma_{r}$ and rms angular spread $\sigma_{r}$. The final emittance ratio is thus simply variable by adjusting the free parameter $\beta=2 p_{o} / e B_{z, c}$, via the magnetic field on the cathode. A first successful demonstration of this method was recently achieved at the A0 experiment at FNAL [15].

In Fig. 6 the beam images at different positions along the beam line of the FNAL experiment are shown. It has been verified that the beam remains flat as it drifts farther downstream, an important experimental achievement that demonstrate the effectiveness of the linear beam optics adapter in the context of RF photoinjector. The measured ratio of emittances is about 100 , with $\varepsilon_{\mathrm{nx}}=41 \mu \mathrm{m}$ and $\varepsilon_{\mathrm{ny}}$ $=0.41 \mu \mathrm{m}$ for a $0.5 \mathrm{nC}$ beam, which yields a geometric mean emittance that is still rather high at $4 \mu \mathrm{m}$. Additional experiments are foreseen in the near future to optimize the emittance compensation process.

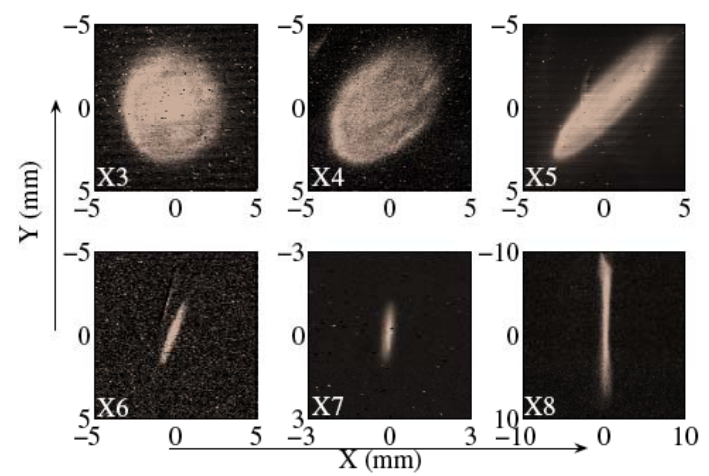

Fig. 6: Measured beam transverse density evolution in the flat beam transformer section.

\section{ACKNOWLEDGEMENT}

This work has been partially supported by the EU Commission in the sixth framework programme, contract no. 011935 EUROFEL 


\section{REFERENCES}

[1] Linac Coherent Light Source (LCLS) Conceptual Design Report, SLAC-R-593 (2002).

[2] TESLA XFEL, Technical Design Report (Supplement), DESY 2002-167, TESLA-FEL 2002-09, (2002).

[3] B. E. Carlsten, Nucl. Instrum. Methods A 285, 313 (1989).

[4] L. Serafini, AIP Conf. Proc. 413, 321 (1997)

[5] O, J, Leuten et al., Phys. Rev. Let, 93, (1997), n. 9

[6] J. B. Rosenzweig et al., "Emittance compensation with dynamically optimized photoelectron beam profiles", Proc. of ERL05, to be published on N.I.M.

[7] L. Serafini, J. B. Rosenzweig, Phys. Rev. E 55 (1997) 7565.

[8] L. Serafini, M. Ferrario, "Velocity Bunching in PhotoInjectors", AIP CP 581, 2001, pag.87.

[9] P. Emma, "Accelerator physics challenges of X-rays FEL SASE sources", Proc. of EPAC-02, Paris 2002.

[10] P. Piot, et al., "Subpicosecond compression by velocity bunching in a photoinjector", Phys. Rev. ST-AB, 6, 033503 (2003).

[11] H. Iijima et al., "Experimental Verification of Velocity Bunching via Shot-by-Shot Measurement at SBand Photoinjector and Linac, submitted to Japanise Journal of Applied Physics

[12] S. G. Anderson et al., "Velocity bunching of highbrightness electron beams", Phys. Rev. ST-AB, 8, 014401 (2005).

[13] L. Merminga, "Technical approaches for highaverage-power free-electron lasers", Rev. Modern Phys., 74, (2002)

[14] R. Brinkmann, Y. Derbenev, and K. Flöttmann "A low emittance, flat-beam electron source for linear colliders", Phys. Rev. ST Accel. Beams 4, 053501 (2001)

[15] Y. E. Sun et al., "Generation of angular-momentumdominated electron beams from a photoinjector", ", Phys. Rev. ST-AB, 7, 123501 (2004).

[16] M. Reiser, "Theory and Design of Charged Particle Beams", J. Wiley \& Sons, (1994).

[17] C. Limborg, Proc. of ERL05, to be published on N.I.M. [18] H. Loos et al., "Temporal e-beam shaping in an SBand accelerator", this conference.

[19] M. Ferrario et al., "HOMDYN Study For The LCLS RF Photo-Injector", Proc. of the $2^{\text {nd }}$ ICFA Adv. Acc. Workshop on "The Physics of High Brightness Beams", UCLA, Nov., 1999, see also SLAC-PUB-8400

[20] L. Serafini et al., "Status of the SPARC Project", this conference.

[21] Y. Kim, "Emittance Damping in TTF2 Booster Linac with Gaussian Longitudinal Laser Beam Profile", this conference.

[22] L. Catani et al., "Design Study of a Movable Emittance Meter Device for the SPARC Photoinjector", Proc. of EPAC 2004

[23] C. Ronsivalle et al., "Optimization of RF compressor in the SPARX injector", this conference.

[24] C. Vaccarezza et al., "Start to end simulation for the SPARX project", this conference.
[25] X.J. Wang et al, Phys. Rev. E 54, R3121 (1996).

[26] D. Janssen, V. Volkov, Nucl. Instr. and Meth. A 452 (2000), 34.

[27] L. Lilje, "Achievement of $35 \mathrm{MV} / \mathrm{m}$ in the TESLA Superconducting Cavities Using Electropolishing as a Surface Treatment", Proc. of EPAC 2004.

[28] T. Srinivasan-Rao et al., "Design, Construction and Status of All Niobium Superconducting Photoinjector at BNL", PAC03, Portland 2003, USA

[29] J. Sekutowicz et al., "Proposed Continuous Wave Energy Recovery Operation of an XFEL", Phys. Rev. ST$\mathrm{AB}, \mathbf{8}, 010701$ (2005).

[30] K. Floettman et al., :Emittance compensation in a superconducting if gun with a magnetic mode", Phys. Rev. ST-AB, 7, 090702 (2004).

[31] I. Ben-Zvi et al., "Ampere Average Current Photoinjector and Energy Recovery Linac", Proc of FEL 2004.

[32] J. Smedley, "Progress on Lead Photocathodes for Superconducting Injectors", this conference.

[33] Ya Derbenev, Adapting Optics for High Energy Electron Collider, UM-HE-98-04, Univ. Of Michigan, 1998. 